Research Article

Shubhra Dwivedi*, Manu Vardhan, and Sarsij Tripathi

\title{
Distributed Denial-of-Service Prediction on IoT Framework by Learning Techniques
}

https://doi.org/10.1515/comp-2020-0009

Received Sep 21, 2019; accepted Jan 02, 2020

\begin{abstract}
Distributed denial-of-service (DDoS) attacks on the Internet of Things (IoT) pose a serious threat to several web-based networks. The intruder's ability to deal with the power of various cooperating devices to instigate an attack makes its administration even more multifaceted. This complexity can be further increased while lots of intruders attempt to overload an attack against a device. To counter and defend against modern DDoS attacks, several effective and powerful techniques have been used in the literature, such as data mining and artificial intelligence for the intrusion detection system (IDS), but they have some limitations. To overcome the existing limitations, in this study, we propose an intrusion detection mechanism that is an integration of a filter-based selection technique and a machine learning algorithm, called information gain-based intrusion detection system (IGIDS). In addition, IGIDS selects the most relevant features from the original IDS datasets that can help to distinguish typical low-speed DDoS attacks and, then, the selected features are passed on to the classifiers, i.e. support vector machine (SVM), decision tree (C4.5), naïve Bayes (NB) and multilayer perceptron (MLP) to detect attacks. The publicly available datasets as KDD Cup 99, CAIDA DDOS Attack 2007, CONFICKER worm, and UNINA traffic traces, are used for our experimental study. From the results of the simulation, it is clear that IGIDS with C4.5 acquires high detection and accuracy with a low false-positive rate.
\end{abstract}

Keywords: Denial-of-service, KDD Cup 99, Support Vector Machine, Machine Learning, Intrusion Detection System

\footnotetext{
^Corresponding Author: Shubhra Dwivedi: Department of Computer Science \& Engineering, NIT Raipur, Chhattisgarh, India; Email: sdwivedi.phd2018.cse@nitrr.ac.in Manu Vardhan, Sarsij Tripathi: Department of Computer Science \& Engineering, NIT Raipur, Chhattisgarh, India
}

\section{Introduction}

In recent years, the Internet of Things (IoT) has attained lots of consideration and provided a vulnerable area of research [1]. IoT-based systems are intricate and contain integrative activities. They are available worldwide, consist of mainly constrained resources and are constructed by loss links. Therefore, crucial modifications of present security concepts for information and wireless networks should be implemented to provide effective IoT security methods. In other words, sustaining the security requirement in a widescale attack surface of the IoT system, is quite a challenging task. However, IoT devices mostly work in an unattended environment. Thus, an intruder can physically contact these devices. Solutions must include broad considerations to fulfill the security prerequisite [2]. Consequently, the security is at a higher risk in IoT systems than in other computing systems, and traditional defense mechanisms, such as encryption, authentication, access control, network security, and Denial of Service (DoS) attacks, are insufficient for extra-large systems with various connected devices, with each part of the system having essential vulnerabilities.

A distributed denial of service (DDoS) attack represents a serious threat to cyberspace security. The destination system or network typically runs out of bandwidth, memory or processing capacity. In general, a DDoS attack is a distributed, large-scale and collaborative attack [3, 4]. It is broadly used in wired and wireless network connections through the internet [5]. Currently, the scale of DDoS attacks on internet security is rising exceptionally. These kinds of attacks may be instigated by intentionally exploiting vulnerabilities of a victim's system (i.e. a host, complete network or router) or overflowing the victim's system using large capacity of network traffic to capture specified resources (such as processor time and memory, and network bandwidth). As a result, the resources accessible to other regular users/customers are restricted or often may not be available. The recent personal victims of the DDoS attack were exposed in [6] and outlines to mitigate the attack have been successfully explored in [7]. Towards the

¿ Open Access. (c) 2020 S. Dwivedi et al., published by De Gruyter. (cc) BY 4.0 License 
defense of DoS attacks, a significant number of works to build IDS systems have been introduced in recent years.

In general, detection schemes are divided into two main categories, namely detection based on misuse and detection based on anomaly [3]. The detection based on misuse depends on a set of signatures avoidance technology by attackers, like signatures to match incoming packages. Systems based on the mechanism of misuse detection can reach high detection rates in known attacks [4]. Nevertheless, they are unable to detect unknown malicious behaviors or even variants of recent attacks. In contrast, the anomaly-based detection schemes employ different detection methods that observe and label any network activity which has substantial deviation from its legitimate traffic profiles as suspicious objects. Because these profiles are based on information on normal network behaviors, the anomaly-based detection schemes can recognize previously unknown attacks. As such, it has opened up an enormous research area for researchers $[5,8]$. In general, effectiveness is measured by detection speed, frequency of false alarms; and efficacy is estimated by response time when an attack occurs. Adding too many features in an anomaly-based IDS doesn't ensure good performance, as it delays the detection engine in making a decision [6]. Therefore, selecting the fewest important features by eliminating irrelevant and redundant features becomes a vital challenge.

Feature selection (FS) is the process of selecting a subset of the original features space based on the ability of discrimination for improving quality and reducing the dimensionality of the dataset [9]. The number of features excerpted from raw data of a network that IDS must observe, is generally high even in case of a small network. To reduce DDoS attacks, several FS methods have been presented and incorporated in IDS that has been classifying the external record into normal or abnormal activities. Overall, there exist two main approaches for choosing significant features, known as wrapper and filter methods. The wrapper methods are executed in an iterative way, in which each execution involves the generation of feature subsets and their evaluation through a classification algorithm. On the other hand, filter method is metrics calculated from statistical and informative theory which reveal the quality of each individual feature without involving any certain classifier [10]. Furthermore, FS is useful for reducing calculation time and facilitating data understanding. In particular, the grouping of features that allows the selection of multiple features at the same time is applicable to the data set with high dimensionality and is advantageous for the creation of efficient IDS [11].
Machine learning is a powerful method of data exploration to learn about between normal and abnormal traffic patterns [12] according to how IoT components and devices interact with one another within the environment. Therefore, for building a robust system, several techniques of insightful machine learning have been widely used, such as multilayer perceptron, decision tree, fuzzy logic, principal component analysis, bayesian network, $k$-nearest neighbor (KNN) [13]. Among these techniques, researchers have found that a decision tree is an encouraging tool in creation of an efficient IDS since it has good performance in terms of efficacy and robustness due to transparency, specificity, integral nature and ease of use [14]. It has the tendency to find more suitable characteristics for the predetermined learning algorithm which translates into higher learning performances, but also tends to require more calculation time and is economically more expensive than the filter model [15].

With the intention of reducing computational costs and improving IDS classification performance, several machine learning techniques with Chi-square (CHI), Information gain (IG) [16] and Correlation-based Feature Selection (CFS) such as filter techniques [17] are used in this study. The filter technique based on the ranking approach sorts the features according to their usefulness in the classification movement. Most classifiers, regardless of the domain of the application, use the ranking strategy to select the subset of final features, in an ad-hoc way, and are widely applied in a previous study [18].

To improve IDS performance, in this article, initial information gain is used as a filter technique to select features, then apply MLP, NB, SVM, and C4.5 as classification algorithms for IDS performance evaluation on the KDD Cup 99, CAIDA DDOS Attack 2007, CONFICKER worm, UNINA traffic traces datasets. It is found that the proposed algorithm (IGIDS) with C4.5 has the finest performance in comparison to other classifiers, attaining high detection rate and accuracy with low false-positive rate. The proposed technique improves the quality of the solution, reduces training time and also improves the efficiency of classifier. The rest of this paper is planned as follows. Section 2 lists some prevailing strategies and reviews their merits and limitations. Section 3 presents the basic knowledge of related techniques. The proposed technique and simulation evaluations are deliberated in sections 4 and 5. Finally, in section 6, the conclusion is discussed. 


\section{Related work}

In this section, we provide a short description of the latest research at the forefront of DDoS, which are widely classified as attacks reducing bandwidth and resources [19]. In a bandwidth-exhaustion attack, intruders flood the victim with huge traffic which averts authentic traffic and intensifies the attack by spreading messages via the broadcast IP address. In a resource depletion attack, intruders aim to connect perilous resources (processor and memory) so that the victim cannot obtain the service. A practical approach to detecting DDoS attacks has been presented by Bojovic et al. [20], who suggested a hybrid technique that integrated detection based on features and volume for the recognition of DDoS attacks. The proposed approach was dependent on an exponential moving-average algorithm to make a decision, employed to both entropy and time series of packet numbers. The performance of the method was estimated by conducting a controlled DDoS experiment in a real academic network.

In the previous studies, it is essential to highlight those including the aspects of DDoS flood intrusions and their measures to counter. In this context, broad-spectrum review of machine learning techniques in different combinations has been used to provide defense against cybercrime. For instance Kumar and Selvakumar [21] evaluated the performance of a comprehensive set of machine learning algorithms for selecting the base classifier using the publicly available KDD Cup dataset. Based on the outcome of the experiments, Resilient Back Propagation (RBP) was chosen as base classifier for this research. The improvement in performance of the RBP classifier was the focus on this study. The proposed classification algorithm (RBPBoost), was achieved by combining an ensemble of classifier outputs and Neyman Pearson cost minimization strategy, for the final classification decision. IoT is one of the fastest developing fields in the history of computing, with an estimated 50 billion devices by the end of 2020. Existing security methods should be enhanced to secure the IoT ecosystem effectively. Machine learning and deep learning (ML/DL) have advanced considerably over the last few years, and machine intelligence has transitioned from laboratory curiosity to practical machinery in several important applications [22].

Creating an intrusion detection system that would work as a realistic, functional model to prevent a DDoS attack is still a challenging issue, as DDoS attacks are varying with time. There have been numerous techniques for DDoS attacks, including filter, wrapper, and hybrid. Tan et al. [23] introduced a DoS attack identification scheme that utilized multivariate correlation analysis (MCA) for the characterization of network traffic accurately by obtaining the geometrical correlations between network traffic attributes. The proposed detection scheme based on MCA was proficient in distinguishing known and unknown DoS attacks efficiently through acknowledgment of patterns of legitimate network traffic only. The performance of this approach was evaluated by utilizing KDD Cup 99 data set. Similarly, to detect and defend against DDoS attacks, Cui et al. [24] have presented the wrapper technique dependent on cognitive-inspired computation with dual address entropy. The flow table attributes of the switch were obtained, and the DDoS attack model was generated by integrating the SVM approach. The proposed technique realized realtime recognition and protection at the preliminary phase of the DDoS attack and restored normal communication in time. The investigation demonstrated that the presented technique identifies attacks fast as well as has a high rate of detection and a low false-positive rate.

Numerous hybrid techniques for identification and defense against DDoS attacks have also been discussed. According to [25], Osanaiye et al. suggested a set-based multifilter selection approach to integrating the output of a fourfilter approach to achieve optimal selection. The experimental evaluation of this technique conducted upon NSLKDD dataset and decision tree classifier is utilized to estimate fitness. Prasad et al. [26] introduced the DDoS detection model based on bio-inspired anomaly for the detection of App-DDoS attacks on the web. The results acquired from the proposed approach have demonstrated the importance and strength of the model to achieve the objectives deliberated for the solution. To detect the DDoS attack, the researchers introduced many detector schemes such as RBF-NN [27], adaptive and hybrid neuro-fuzzy systems [17], distributed state estimation methods based on ADMM [28], adaptive hybrid IDS [19].

Unlike the previous reported techniques, this article introduces an approach that helps with data mining in security defense from DDoS attacks using a minimum set of features that integrates the IG technique as filter and C4.5 that is found to be the best classification technique to obtain the optimal subset of features used to accurately classify DDoS attacks on KDD Cup 99, CAIDA DDOS Attack 2007, CONFICKER worm, and UNINA traffic traces datasets. 


\section{Background}

\subsection{Information Gain}

Information gain is based on the entropy of the system which estimates the uncertainty of a random variable [25]. It is predominantly employed in feature selection as filter to rank attributes with respect to their IG value. The IG value of each attribute signifies its importance to the datasets, i.e., a higher IG value shows that this attribute donates more information. Assume $s$ is a discrete random variable along with two possible outcomes. The binary entropy function $H$, designated in a logarithmic base 2, is denoted via Eq. (1):

$$
H(s)=-p(+) \log _{2} p(+)-p(-) \log _{2}(p(-))
$$

where $(+,-)$ are types of classes, $p(+)$ signifies the probability of a sample $s \in(+)$, and $p(-)$ represents the probability of a sample $s \in(-)$. Entropy enumerates the uncertainty of each attribute while making a decision. Eq. (2) computes the conditional entropy of two events $R$ and $S$, while $R$ has value $r$ :

$$
\begin{aligned}
H\left(\frac{S}{R}\right) & =\sum_{r \in R} p_{r}(r) H\left(\frac{S}{R}=r\right) \\
& =-\sum_{r \in R} p_{r}(r) \sum_{s \in S} p\left(\frac{S}{r}\right) \log _{2} p_{s}\left(\frac{s}{r}\right) \\
& =-\sum_{r \in R} \sum_{s \in S} p_{r s}(r, s) \log _{2} p_{s}\left(\frac{s}{r}\right)
\end{aligned}
$$

Where $\lim _{r \rightarrow 0} r \log _{2} r=0$

The error of entropy and incorrect classification is maximum while the distribution of a class is uniform. The minimum entropy value is reached as long as all the samples relate to the same class. IG is extensively utilized on highdimensional data to measure the effectiveness of the attributes in the classification. It is the expected amount of information or the reduction of entropy. That is, the gain of information from function $r$ is calculated by Eq. (3):

$$
I G(s, r)=H(s)-H\left(\frac{s}{r}\right)
$$

The primary idea behind this technique is to perform ranking on the subsets of attributes by computing the value of IG for each attribute in a decreasing order. Each attribute obtains a score between 1 (more significant) and 0 (less significant). The attributes having maximum scores are considered as the minimum set of input attributes for the next phase. Larger information gain demonstrates greater discriminatory power for making the decision.
Example: Let IDS dataset $\boldsymbol{F}=\left\{\boldsymbol{f}_{\mathbf{1}}, \boldsymbol{f}_{\mathbf{2}}, \boldsymbol{f}_{\mathbf{3}}\right\}$ be a set of three features i.e., scr_bytes $\left(\boldsymbol{f}_{\mathbf{1}}\right)$, diff srv rate $\left(\boldsymbol{f}_{\mathbf{2}}\right)$, and dst host srv count $\left(\boldsymbol{f}_{3}\right)$. The main objective is to compute highly correlated features for each feature present in the dataset. First, we compute feature-class mutual information (MI) for each feature and select the feature that has the maximum value. Feature $\boldsymbol{f}_{\mathbf{1}}$ has the highest value and hence, $\boldsymbol{f}_{\mathbf{3}}$ is removed from $F$ and is put in the optimal feature set, say, $F^{\prime}$. Next, for feature $\boldsymbol{f}_{\boldsymbol{j}} \in F$, we compute feature-feature MI with each feature $\boldsymbol{f}_{\boldsymbol{i}} \in F^{\prime}$ and store the average value of feature-feature MI for $\boldsymbol{f}_{\boldsymbol{j}}$. This way, we compute featurefeature MI for $\boldsymbol{f}_{\mathbf{1}}$ and $\boldsymbol{f}_{\mathbf{2}}$. Again, we compute feature-class MI for $\boldsymbol{f}_{\mathbf{1}}$ and $\boldsymbol{f}_{\mathbf{2}}$. This procedure is continued until we get a subset of $k$ features.

$$
\begin{aligned}
& I G\left(y ; f_{1}\right)=0.11, \quad I G\left(y ; f_{2}\right)=0.04, \\
& I\left(y ; f_{3}\right)=0.18 \quad I G\left(f_{1} ; f_{2}\right)=1.15, \\
& I G\left(f_{1} ; f_{3}\right)=1.28, \quad I G\left(f_{2} ; f_{3}\right)=0.57 \\
& I G\left(y ; \frac{f_{1}}{f_{3}}\right)=0.43, \quad I G\left(y ; \frac{f_{2}}{f_{3}}\right)=0.31
\end{aligned}
$$

Definition 1: Feature relevance: Let $S$ be a full set of features, $\boldsymbol{f}_{\mathrm{i}}$ feature and $\boldsymbol{f}_{\mathrm{i}}=\boldsymbol{S}-\left\{\boldsymbol{f}_{\mathrm{i}}\right\}$. Feature $\boldsymbol{f}_{\mathrm{i}}$ is strongly relevant iff $\boldsymbol{I}\left(\boldsymbol{c} \mid \boldsymbol{f}_{\mathbf{i}}, \boldsymbol{f}_{\mathbf{j}}\right) \neq \boldsymbol{I}\left(\boldsymbol{c} \boldsymbol{f}_{\mathbf{j}}\right)$ otherwise if $\boldsymbol{I}\left(\boldsymbol{c} \mid \boldsymbol{f}_{\mathbf{i}}, \boldsymbol{f}_{\mathbf{j}}\right) \cong$ $\boldsymbol{I}\left(\boldsymbol{c} \mid \boldsymbol{f}_{\mathrm{j}}\right)$ then $\boldsymbol{f}_{\mathrm{i}}$ is weakly relevant to the class c.

Definition 2: Feature-class relevance: It is defined as the degree of feature-class MI for a given class $\boldsymbol{c}_{\mathbf{i}}$ of a given dataset $D$, where data elements described by $d$ features. A feature $\boldsymbol{f}_{\mathbf{i}} \in F^{\prime}$, is an optimal subset of relevant features for $\boldsymbol{c}_{\mathbf{i}}$, if the relevance of $\left(\boldsymbol{f}_{\mathbf{i}}, \boldsymbol{c}_{\mathbf{i}}\right)$ is high.

Definition 3: Relevance score: The relevance score of each feature $f_{\mathrm{i}}$ is the degree of relevance in terms of correlation between feature and class label. Based on this value, a rank can be assigned to each feature $\boldsymbol{f}_{\mathrm{i}}$, where $\{i=$ $1,2,3, \cdots, d\}$. For a given feature $\boldsymbol{f}_{\mathbf{i}} \in F^{\prime}$, the relevance score is high.

\subsection{Support Vector Machine}

SVM is a machine learning technique to facilitate categorization between two groups. In this, a hyperplane is formed to differentiate between positive and negative samples considering the principle of structural risk minimization [29]. An anticipated characteristic of SVM is that this carries out the classification utilizing support vectors instead of the whole data set, and therefore this is enormously robust for outliers and predicts effectively.

Let $M$ represent the training data points $\left\{\left(x_{1}, y_{1}\right),\left(x_{2}, y_{2}\right), \ldots,\left(x_{M}, y_{M}\right)\right\}$, where $x_{i} \in R^{d}$ and 
$y_{i} \in\{+1,-1\}$. Each data point contains similar Lagrangian multiplier $\beta_{i}$ allocating comparative weight. When the hyperplane is determined as $(w, c)$, the prediction of class for data point $x$ is calculated as in Eq. (4)

$$
\begin{aligned}
F(x) & =\operatorname{sgn}\left(w, k\left(x, x_{i}\right)-c\right) \\
& =\operatorname{sgn}\left\{\sum_{i=1}^{M} \beta_{i} y_{i} e^{\left(\frac{\left\|x-x_{i}\right\|^{2}}{2 \sigma^{2}}\right)}-c\right\}
\end{aligned}
$$

where $s g n$ represents the sign function, $k(\cdot, \cdot)$ shows the radial basis kernel function, $w$ represents weight, $x$ shows a data point in the input space, $\sigma$ depicts standard deviation, and $c$ represents bias. When the hyperplane is welldefined, all the points positioned nearby contain $\beta_{i}>0$, and are support vectors. In addition, the residual points exhibit $\beta_{i}=0$. SVM has been implemented effectively for resolving several issues in real scenarios, including image classification and bioinformatics.

\subsection{Multi-layer Perceptron}

MLP is a kind of artificial neural network having minimum of three layers of neurons, known as an input layer, one or more hidden layers, and an output layer [3]. The basic approach of this method is that it maps many real-valued inputs into outputs by altering the weight amongst its internal nodes. MLP acquires a function $f(x): \boldsymbol{R}^{i} \rightarrow \boldsymbol{R}^{t}$ while training of a dataset, utilizing back-propagation learning approach [3], where $i, t \in Q^{+}$represents dimensions of input and output individually. This is calculated as in Eq. (5)

$$
y=\delta\left\{\sum_{i=1}^{m}\left(w_{i} X+b\right)\right\}=\delta\left(W^{T} X+b\right)
$$

where $\delta$ shows the activation function, $w$ indicates the weight vectors, $X$ denotes the input vectors, and $b$ signifies the bias. Practically, the neural classifier has been extensively implemented in several fields like pattern classification, recognition, and prediction.

\subsection{Naïve Bayes}

Naive Bayes is a Bayesian network technique that considers that all attributes are independent [30]. The basic NB classification approach is dependent on the Bayes rule theorem to look for the maximum probability hypothesis which recognizes the label of class. It is employed to make a prediction for data points utilizing the maximum back function (MAP) calculated as in Eq. (6)

$$
P(L O)=\operatorname{argmax}_{w \in(1,2, \ldots M)} P\left(L_{w}\right) \prod_{j=1}^{M} P\left(O_{j} L_{w}\right)
$$

where $L$ represents the class label, $O$ displays the observation of each class, $w$ depicts the class number, $P(L O)$ demonstrates the probability of a given class and $\prod_{j=1}^{M} P\left(O_{j} \mid L_{w}\right)$ presents the multiplication of the probabilities of all instances subjected to their classes to obtain the maximum output.

\subsection{Decision Tree}

A decision tree is a structural approach akin to a tree, which comprises leaves and branches [31]. The leaves signify class labels, whereas the branches indicate attributes that result in the classification of data. The decision rules are determined by if-then rules for classification of data entries. These rules are utilized specifically to solve attribute selection problems of various types (for example, strings and real numbers). A decision tree is built using divideand-conquer schemes. As demonstrated in Eqs. (7)-(8), the Gain Ratio is a measure of the decision tree to estimate the performance. Assume that there is a training dataset $D$, the expected information required to accurately categorize an attack in IDS, $x_{j} \in D$ is calculated as:

$$
\begin{aligned}
& \text { Entropy }=-\sum_{j=1}^{m} p_{j} \log \left(p_{j}\right) \\
& \text { GainRatio }=\frac{\operatorname{Gain}(p)}{\operatorname{SplitInfo}(p)}
\end{aligned}
$$

The function Split info is presented in Eq. (9).

$$
\text { SplitInfo }(p, \text { test })=-\sum_{j=1}^{m} p\left(\frac{j}{p}\right) \log \left(p\left(\frac{j}{p}\right)\right)
$$

where $p$ represents probability distribution of data, whereas in log, two is used as base, due to estimate information as bit.

\section{Proposed Method}

In this study, a hybrid technique in investigated by combining IG as a filter technique for reducing dimensions with various classifiers such as SVM, C4.5, NB, and MLP approaches. From Figure 1, we observed that the detection schemes of our approach contain three steps: comprising IG as a filter feature selection technique to choose 


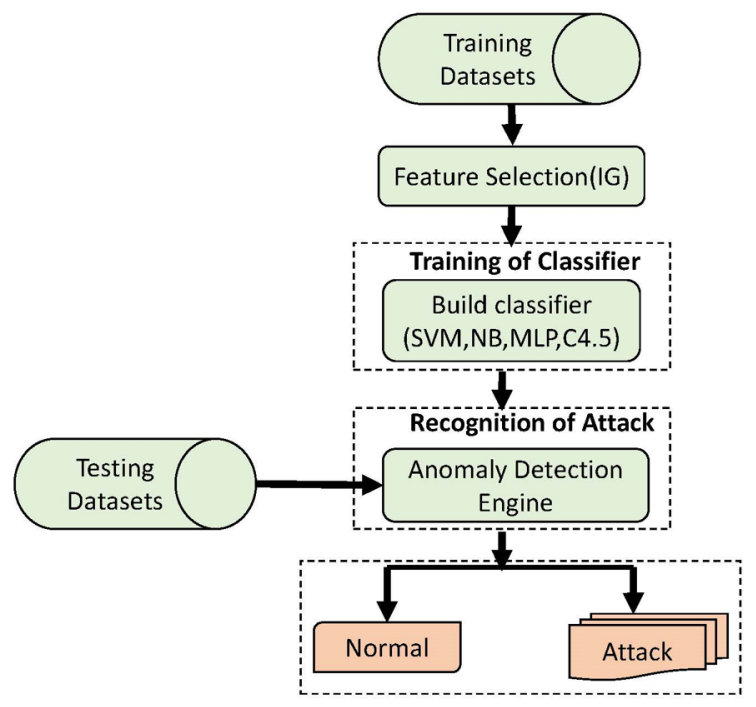

Figure 1: The framework of the proposed IGIDS method.

the relevant features reducing the dimensionality, training the classifier utilizing SVM, C4.5, NB and MLP approaches, and performing the identification of attacks. Among these classifiers, C4.5 is the best classifier based on performance metrics to detect intrusions. The proposed system has acquired the best performance concerning accuracy, detection rate, precision, F-measure, specificity, false-positive rate (FPR) and AUC.

\subsection{Data Pre-processing}

Normalization is done on each feature of the input data matrix so as to provide the same weight or priority to all features. Preprocessing aims to eliminate redundant and irrelevant features in the samples. When generating the subset of features, we choose a suitable minimum set of features demonstrating differences between the classes for the following efficient classification. Here, IG is used to decrease the dimensionality of data sets as a filter technique [10]. In this paper, the filter approach is used due to its simplicity and a faster processing speed compared to other approaches. Therefore, IG for a huge number of attributes is estimated for data sets. Finally, for the next step, minimal numbers of attributes with high information gains are calculated.

\subsection{Classification Technique}

In this article, KDD Cup 99, CAIDA DDOS Attack 2007, CONFICKER worm, and UNINA traffic traces datasets are em- ployed for experiments, KDD Cup 99 is one of the most popular data sets for intruder detection.

In the fields of intrusion detection, classification is the process of deciding to which group it belongs in a given, previously unknown, sample, i.e., a function that determines a class label to the instances designated by a set of attributes. The proposed method can improve the performance of intruder detection, therefore, we have employed a cross-validation procedure to ensure that the classifier generalizes the unseen data well. In this work, we employ four classifiers, that is, NB, SVM, C4.5, and MLP. When the optimal subset of attributes is selected by the IG-based filter feature selection technique, this subset is then passed to the training phase of the classifier where different SVM, NB, MLP, and C 4.5 classifiers are used in which C 4.5 works well compared to other techniques in discriminating one class of records from others. For instance, the classifier of the normal class differentiates normal data from attack data (all types of attacks exist in DDoS). The pseudo-code of the proposed method is provided in Algorithm 1.

Algorithm 1: Pseudo code of Proposed method (IGIDS)

Input: Dataset D has $R\left\{r_{j}, j=1,2, \ldots N\right\}$ features and $k$ instances, $\varphi$ : the number of classifiers, $n$ : the number of features selected, $Z$ : reduced datasets with the $Y$ training set and the $X$ testing set

Begin

Procedure Compute_IG $(s, r)$

Compute information required to classify a given instance according to Eq. (1)

While $1 \leq j \leq k$ do

Estimate conditional entropy for attribute $r_{j}$ according to Eq. (2)

Estimate information gain for each attribute $r_{j}$ according to Eq. (3)

$Z \leftarrow$ the $n$ attributes with the highest value of scores return $Z$

For $i=1: \varphi$ do

randomly separate utilizing 10 -fold cross-validation training set $Y$ with $Z$

test $\varphi$ classifiers using testing set $X$

estimate performance of the classifier

End For

End While

Return Accuracy, F-measure, Precision, FPR, Sensitivity, and AUC 


\section{Results and Analysis}

In this section, we analyze the results of the simulation of the IDS technique based on information gain (IGIDS) to determine its effectiveness in the detection of DDoS attacks. Results are also obtained in comparison with the result of other filter-based techniques such as CFS (correlationbased feature selection), Relief-F, Chi-square together with different classifiers such as MLP, NB, C4.5, and SVM. The classification performance of the proposed approach on the KDD Cup 99, CAIDA DDOS Attack 2007, CONFICKER worm, and UNINA traffic traces datasets is evaluated using a 10 -fold CV approach. The used datasets have been extensively employed in the domain of intrusion detection research and utilized in several recent investigations. They have been adopted to evaluate the performance of an IDS based on anomalies in the detection of new intrusions. The analysis of our approach based on the datasets provides convincing and comparative evaluations using other related cutting-edge techniques.

\subsection{Performance Measures}

We estimate the performance of our approach by using seven performance parameters, i.e., accuracy, sensitivity, specificity, precision, f-measure, AUC (Area under curve) and false positive rate [2]. These performance metrics are determined as:

a) Accuracy $=\frac{T N+T P}{T P+T N+F P+F N}$

b) Recall $(R e)=$ Sensitivity $=$ Detection Rate $(D R)=$ $\frac{T P}{T P+F N}$

c) Precision $(\mathrm{Pr})=\frac{T P}{T P+F P}$

d) $F-$ measure $=\frac{2 \star P r+R e}{P r+R e}$

e) False positive rate $(F P R)=\frac{F P}{F P+T N}$

f) Specificity $=1-F P R$

g) $A U C=\frac{(\text { Sensitivity }+ \text { Specificity })}{2}$

Here, $F P, F N, T P$, and $T N$, are false positive, false negative, true positive and true negative in the independent datasets.

\subsection{Description of Dataset}

In this study, KDD Cup 99, CAIDA DDOS Attack 2007, CONFICKER worm, and UNINA traffic traces datasets are employed [17, 32]. Firstly, all the network traffic records are extracted from the above-mentioned datasets. In KDD Cup 99 dataset, the 22 types of attacks are not distributed equally. In this article, we only focus on the DDoS attacks [32].
CAIDA DDoS Attack 2007 dataset contains approximately one hour of unusual traffic traces from DDoS attacks. This type of denial-of-service attack attempts to block contact to the targeted system by overwhelming computing capacity of the system and by consuming all of the bandwidth of the network connecting the system to the internet. Conficker collected data from UCSD Network Telescope based on three days of network study in between November 2008 and January 2009. The first day covers the onset of the Conficker A infection. On the second day, only Conficker A was active, and during the third and final day both Conficker A and B were active. The dataset contains $68 \mathrm{com}$ pressed pcap files each containing one hour of traces. UNINA dataset denotes traffic traces taken by passively monitoring incoming traffic at WAN access router at the University of Napoli Federico II. D-ITG is traffic group and active measurement software architecture which imitates many network traffic profiles and performs packet-level capacities. This helps to prevent hidden bias in consecutive data that affects the generation of normal profile and attack identification through our approach.

\subsection{Simulation Results and Discussion}

In this section, we evaluate the performance of the proposed approach with the cutting-edge methods implemented in the generation of intrusion detection systems. In this context, we use three different filter-based techniques, such as CFS, Relief-F and Chi-square together with different SVM, NB, MLP, and C4.5 classifiers, comparing the performance of IDS based on information gain for DDoS attack identification. Table 1 shows the comparative analysis of different filter-based feature selection techniques along with different classifiers on four datasets. From Table 1, we observe that the information gain works together with $\mathrm{C} 4.5$, which has shown better performance than other techniques in terms of $99.25 \%$ accuracy, $98.80 \%$ detection rate, and $98.24 \%$ precision in the identification of DDoS attacks on KDD Cup 99 data, as well as $98.35 \%$ accuracy, $98.12 \%$ detection rate, and $98.45 \%$ precision on CONFICKER worm dataset.

Figure 2 illustrates the comparison of the proposed approach with other filter techniques such as CFS, Chisquare, Relief-F concerning computational time and detection rate. From Figure 2(a), we observed that the proposed technique take less time in comparison to other technologies with C4.5, whereas Figure 2(b) demonstrates that IG with C4.5 classifier attains high detection rate of $98.80 \%$ in contrast to other methods on KDD Cup 99 dataset. 
Table 1: Comparative performance of the proposed method with other techniques.

\begin{tabular}{|c|c|c|c|c|c|c|c|c|c|c|}
\hline \multicolumn{6}{|c|}{ KDD Cup 99} & \multicolumn{5}{|c|}{ CAIDA DDOS Attack 2007} \\
\hline Measures & MLP & NB & SVM & C4.5 & Method & Measures & MLP & NB & SVM & C4.5 \\
\hline Accuracy & 91.77 & 68.80 & 97.64 & 98.75 & CFS & Accuracy & 90.23 & 70.20 & 95.14 & 96.35 \\
\hline Detection rate & 90.80 & 67.56 & 97.56 & 98.39 & & Detection rate & 90.46 & 69.36 & 94.66 & 95.89 \\
\hline Precision & 91.60 & 71.00 & 97.53 & 98.22 & & Precision & 91.49 & 72.43 & 94.21 & 95.38 \\
\hline Accuracy & 91.94 & 67.51 & 97.41 & 98.89 & Relief & Accuracy & 91.36 & 68.27 & 94.93 & 95.46 \\
\hline Detection rate & 90.64 & 67.80 & 97.24 & 98.20 & & Detection rate & 91.52 & 69.80 & 95.20 & 95.54 \\
\hline Precision & 91.47 & 71.63 & 97.48 & 98.33 & & Precision & 91.47 & 70.24 & 95.32 & 94.25 \\
\hline Accuracy & 87.35 & 68.35 & 98.52 & 98.54 & Chi-square & Accuracy & 88.52 & 69.45 & 96.31 & 96.37 \\
\hline Detection rate & 87.40 & 67.56 & 97.63 & 98.35 & & Detection rate & 89.47 & 70.36 & 96.29 & 96.56 \\
\hline Precision & 87.2 & 71.82 & 97.84 & 98.47 & & Precision & 86.72 & 71.34 & 95.64 & 96.65 \\
\hline Accuracy & 88.57 & 69.84 & 98.27 & 99.25 & IG & Accuracy & 85.34 & 70.14 & 96.27 & 97.80 \\
\hline Detection rate & 87.68 & 68.42 & 97.45 & 98.80 & & Detection rate & 86.25 & 69.56 & 95.72 & 97.45 \\
\hline Pre & 87.94 & 71.33 & 97.6 & 98.24 & & ision & 86.74 & 70.39 & 96.40 & 97.18 \\
\hline \multicolumn{6}{|c|}{ CONFICKER worm } & \multicolumn{5}{|c|}{ UNINA traffic traces } \\
\hline Measures & MLP & NB & SVM & C4.5 & Method & Ires & MLP & NB & SVM & C4.5 \\
\hline Accuracy & 91.53 & 69.93 & 96.31 & 97.45 & CFS & acy & 89.34 & 69.60 & 95.72 & 96.42 \\
\hline Detection rate & 90.62 & 68.54 & 95.68 & 97.30 & & Dete & 90.36 & 68.49 & 94.26 & 96.19 \\
\hline Precision & 89.97 & 70.58 & 96.42 & 97.48 & & Precis & 90.46 & 70.43 & 95.41 & 96.21 \\
\hline Accuracy & 89.68 & 72.31 & 97.25 & 97.69 & Relief & Accuracy & 90.64 & 69.21 & 96.52 & 96.32 \\
\hline Detection rate & 90.64 & 70.08 & 97.54 & 97.41 & & Detection rate & 89.52 & 68.45 & 96.34 & 96.29 \\
\hline Precision & 91.72 & 70.65 & 97.49 & 97.67 & & & 90.27 & 70.39 & 96.49 & 96.38 \\
\hline Accuracy & 86.76 & 68.95 & 96.49 & 97.81 & Chi-square & Accuracy & 88.32 & 67.15 & 95.32 & 96.34 \\
\hline Detection rate & 86.40 & 68.29 & 95.73 & 97.24 & & Detection rate & 87.57 & 67.46 & 95.27 & 96.29 \\
\hline Precision & 87.63 & 70.39 & 96.71 & 97.46 & & Precision & 89.20 & 68.92 & 95.64 & 96.41 \\
\hline Accuracy & 89.38 & 72.37 & 97.34 & 98.35 & IG & Accuracy & 89.46 & 70.24 & 96.17 & 96.63 \\
\hline Detection rate & 88.61 & 70.62 & 97.25 & 98.12 & & Detection rate & 88.57 & 69.35 & 96.52 & 96.45 \\
\hline Precision & 89.79 & 71.52 & 97.81 & 98.45 & & Precision & 89.73 & 71.47 & 96.49 & 96.72 \\
\hline
\end{tabular}

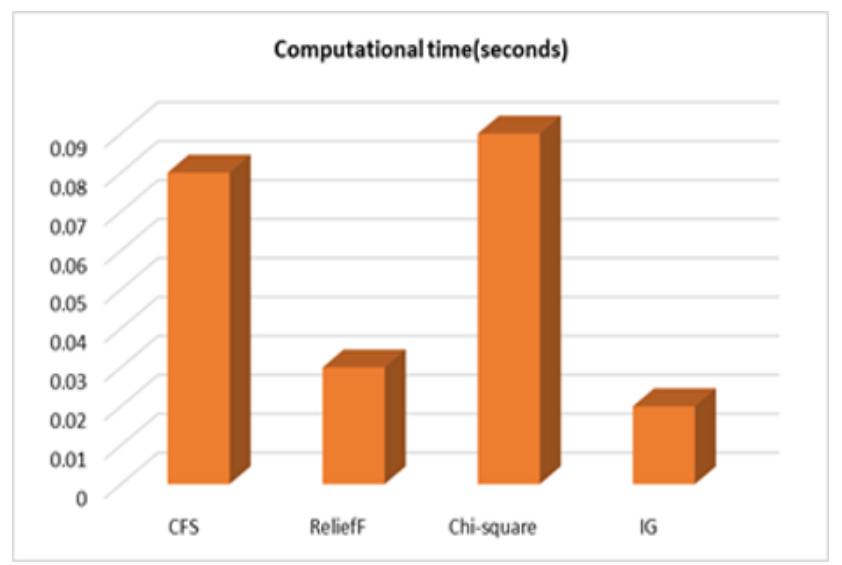

(a)

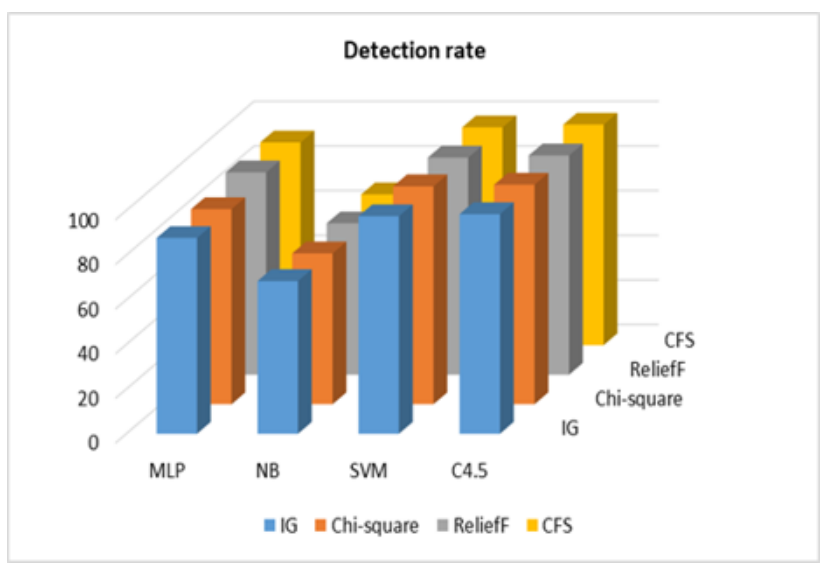

(b)

Figure 2: (a)-(b) Comparative analysis of IG with respect to other filter techniques in terms of computational time and detection rate. 
Table 2: Evaluation performance of used datasets.

\begin{tabular}{|c|c|c|c|c|c|c|c|c|c|c|}
\hline \multicolumn{6}{|c|}{ KDD Cup 99} & \multicolumn{5}{|c|}{ CAIDA DDOS Attack 2007} \\
\hline Measures & MLP & NB & SVM & C4.5 & Method & Measures & MLP & NB & SVM & C4.5 \\
\hline FPR & 18.00 & 64.30 & 3.20 & 3.05 & CFS & FPR & 19 & 65.42 & 4.6 & 4.35 \\
\hline F-measure & 90.40 & 56.40 & 97.60 & 98.10 & & F-measure & 89.40 & 57.46 & 96.44 & 97.21 \\
\hline AUC & 89.90 & 84.70 & 97.20 & 98.20 & & AUC & 88.75 & 85.60 & 96.30 & 97.16 \\
\hline FPR & 17.62 & 65.44 & 3.29 & 3.10 & ReliefF & FPR & 18.31 & 67.47 & 4.32 & 4.20 \\
\hline F-measure & 91.46 & 57.36 & 98.41 & 98.41 & & F-measure & 90.57 & 58.16 & 97.29 & 97.35 \\
\hline AUC & 89.80 & 85.79 & 97.36 & 98.34 & & AUC & 88.64 & 84.57 & 96.29 & 97.19 \\
\hline FPR & 19.10 & 64.75 & 3.13 & 3.08 & Chi-square & FPR & 20.12 & 65.48 & 4.36 & 4.29 \\
\hline F-measure & 87.10 & 56.49 & 97.48 & 98.46 & & F-measure & 87.19 & 66.34 & 96.22 & 97.24 \\
\hline AUC & 89.60 & 84.96 & 97.15 & 98.56 & & AUC & 88.56 & 83.71 & 96.52 & 97.31 \\
\hline FPR & 18.05 & 63.21 & 3.05 & 3.01 & IG & FPR & 19.47 & 64.29 & 4.36 & 4.23 \\
\hline F-measure & 87.53 & 56.47 & 97.60 & 98.53 & & F-measure & 86.42 & 55.62 & 96.54 & 97.26 \\
\hline AUC & 90.60 & 84.93 & 97.53 & 98.79 & & AUC & 89.25 & 85.37 & 96.28 & 97.42 \\
\hline \multicolumn{6}{|c|}{ CONFICKER worm } & \multicolumn{5}{|c|}{ UNINA traffic traces } \\
\hline Measures & MLP & NB & SVM & C4.5 & Method & Measures & MLP & NB & SVM & C4.5 \\
\hline FPR & 21 & 66.45 & 5.24 & 4.46 & CFS & FPR & 20.53 & 67.53 & 4.56 & 4.75 \\
\hline F-measure & 88.72 & 58.42 & 95.26 & 96.45 & & F-measure & 89.58 & 54.64 & 95.21 & 96.34 \\
\hline AUC & 90.78 & 83.56 & 95.47 & 96.29 & & AUC & 87.34 & 83.82 & 95.36 & 96.12 \\
\hline FPR & 18.39 & 67.48 & 4.69 & 4.28 & ReliefF & FPR & 18.41 & 68.24 & 4.35 & 4.58 \\
\hline F-measure & 90.79 & 55.76 & 95.61 & 96.53 & & F-measure & 90.26 & 55.29 & 95.35 & 96.41 \\
\hline AUC & 88.56 & 84.48 & 95.75 & 96.34 & & AUC & 89.8 & 85.79 & 95.16 & 96.34 \\
\hline FPR & 20.09 & 66.15 & 4.41 & 4.38 & Chi-square & FPR & 20.25 & 66.49 & 4.27 & 4.38 \\
\hline F-measure & 85.16 & 56.21 & 95.84 & 96.78 & & F-measure & 85.34 & 54.78 & 95.76 & 96.36 \\
\hline AUC & 87.49 & 82.97 & 95.58 & 96.65 & & AUC & 86.41 & 83.86 & 95.54 & 96.42 \\
\hline FPR & 19.73 & 65.31 & 4.07 & 4.16 & IG & FPR & 19.28 & 65.27 & 4.47 & 4.42 \\
\hline F-measure & 86.19 & 54.25 & 95.74 & 97.43 & & F-measure & 85.52 & 55.32 & 95.68 & 96.72 \\
\hline AUC & 89.54 & 83.51 & 96.07 & 97.57 & & AUC & 90.54 & 83.62 & 95.21 & 96.49 \\
\hline
\end{tabular}

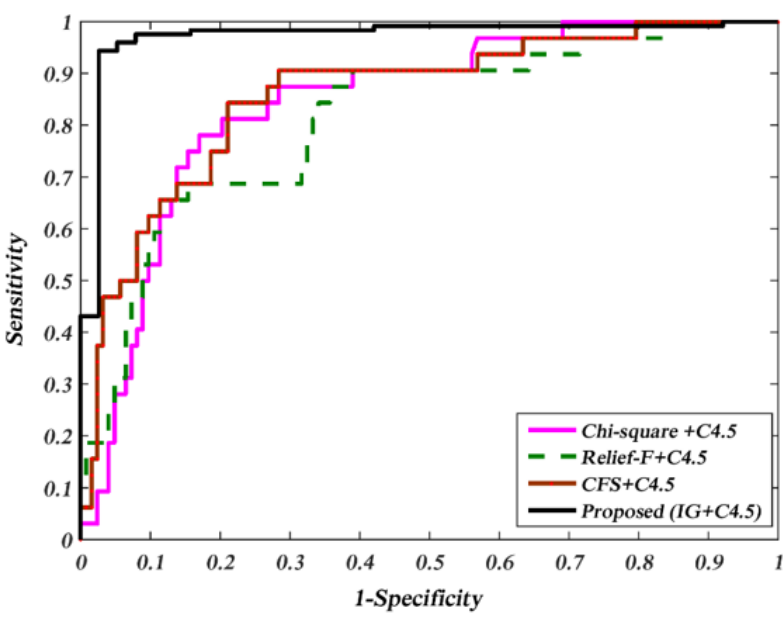

Figure 3: The ROC curves of KDD Cup 99 data set compared to other filter techniques with C4.5.
In Table 2, the performance of the proposed method based on the selected features is determined concerning the F-measure, AUC, and FPR with different other filterbased techniques. According to the achieved results, the proposed technique confirms its efficacy in the detection of DDoS attacks based on the performance metrics, i.e. 3.01\% FPR, 98.53\% F-measure, 98.79\% AUC and 4.16\% FPR, 97.43\% F-measure, 97.57\% AUC, utilizing KDD Cup 99 and CONFICKER worm datasets, respectively.

The comparative result shows that using a filter-based feature selection technique with Decision tree, we obtain the best accuracy of $99.25 \%, 98.80 \%$ detection rate, 3.01\% false-positive rate. Therefore, the hybrid technique proposed in this paper is effective for DDoS detection in comparison to other reported literature. From Figure 3, we clearly see that the proposed method outperforms other methods. 


\section{Conclusion}

The ubiquity of internet connectivity has led to considerable increase in cyber-crime activity and intrusions. Detection of attacks on IoT-based environment has become more complex, because different physical devices and wireless transmission to mobile and cloud architectures, must be protected and combined with other technologies. The volume of information is the most appreciated component for both organizations and users, and DDoS attacks cause a boundless risk in the network. The evolution of various emerging machine learning technologies is one of the important ways that allows us to improve the security of the IoT and to prevent different DDoS attacks. The current countermeasures make DDoS identification exceptionally problematic, therefore numerous other techniques have been introduced to resolve this problem. On the other hand, existing approaches are still posing issues, therefore, a new technique is needed for more effective detection and prevention of DDoS attacks. To deal with these limitations, in this paper, we have designed an information gain based IDS method, called IGIDS. This approach is performed in two steps: firstly, the features are selected through information gain and then the selected features are passed to the different classifiers SVM, NB, MLP, and C4.5 to classify the DDoS attack. It has been found that the filter technique information gain with the C4.5 classifier has better performance than other prevailing techniques with respect to the accuracy, detection, and false-positive rate to identify DDoS attacks in KDD Cup 99 dataset.

\section{References}

[1] Buczak A., and Guven E., A survey of data mining and machine learning methods for cyber security intrusion detection, IEEE Commun Surv Tutorials, 2015, 18(2), 1153-75

[2] Chaabouni N., Mosbah M., Zemmari A., Sauvignac C., Faruki P., Network Intrusion Detection for loT Security Based on Learning Techniques, IEEE Commun Surv Tutorials, 2019, 21(3), 2671-701

[3] Zargar S.T., Joshi J., Tipper D., A survey of defense mechanisms against distributed denial of service (DDOS) flooding attacks, IEEE Commun. Surv. Tutorials, 2013, 15, 2046-2069

[4] Sagar K., Sanjaya S., Panda K., and Sahoo S. Toward secure software-defined networks against distributed denial of service attack, The Journal of Supercomputing, 2019, 1-46

[5] Praseed A., Santhi Thilagam P., DDoS attacks at the application layer: Challenges and research perspectives for safeguarding web applications, IEEE Commun Surv Tutorials, 2019, 21(1), 66185

[6] Daniel Marcillo Lara P., Alejandro Maldonado-Ruiz D., Daniel Arrais Díaz S., Isabel Barona López L., Leonardo Valdivieso
Caraguay Á., Trends on Computer Security: Cryptography, User Authentication, Denial of Service and Intrusion Detection, ArXiv e-prints, 2019

[7] Toledo A.L., Wang X., Robust detection of MAC layer denial-ofservice attacks in CSMA/CA wireless networks, IEEE Trans Inf Forensics Secur, 2008, 3(3), 347-58

[8] Sahoo K.S., Puthal D., Tiwary M., Rodrigues J.J.P.C., Sahoo B., and Dash R., An Early Detection of Low Rate DDoS Attack to SDN Based Data Center Networks using Information Distance Metrics, Futur. Gener. Comput. Syst., 2018

[9] Shukla A.K., Identification of cancerous gene groups from microarray data by employing adaptive genetic and support vector machine technique, Comput Intell., 2019, 1-30

[10] Shukla A.K., Tripathi D., Reddy B.R., Chandramohan D., A study on metaheuristics approaches for gene selection in microarray data: algorithms, applications and open challenges, Evol. Intell, 2019, 1-21

[11] Shukla A.K., Singh P., Vardhan M., A new hybrid wrapper TLBO and SA with SVM approach for gene expression data, Information Sciences, 2019, 503, 238-254

[12] Bhuvaneswari B.A., Selvakumar S., Deep Radial Intelligence with Cumulative Incarnation approach for detecting Denial of Service attacks, Neurocomputing, 2019, 340, 294-308

[13] Singh K.J., Thongam K., De T., Detection and differentiation of application layer DDoS attack from flash events using fuzzy-GA computation, IET Inf Secur, 2018, 12(6), 502-12

[14] Lee S.J., Xu Z., Li T., Yang Y., A novel bagging C4.5 algorithm based on wrapper feature selection for supporting wise clinical decision making, J Biomed Inform, 2018, 78, 144-55

[15] Nakano M., Takahashi A., Takahashi S., Fuzzy logic-based portfolio selection with particle filtering and anomaly detection, Knowledge-Based Syst, 2017, 131, 113-24

[16] Wang W., He Y., Liu J., Gombault S., Constructing important features from massive network traffic for lightweight intrusion detection, IET Inf Secur, 2015, 9(6), 374-9

[17] Arun Raj Kumar P., Selvakumar S., Detection of distributed denial of service attacks using an ensemble of adaptive and hybrid neuro-fuzzy systems, Comput Commun, 2013, 36(3), 303-19

[18] Selvakumar B., Muneeswaran K., Firefly algorithm based Feature Selection for Network Intrusion Detection, Comput Secur, 2018

[19] Shukla A.K., Singh P., Vardhan M., A hybrid framework for optimal feature subset selection, Journal of Intelligent \& Fuzzy Systems, 2019, 36, 2247-2259

[20] Bojović P.D., Bašičević I., Ocovaj S., Popović M., A practical approach to detection of distributed denial-of-service attacks using a hybrid detection method, Comput Electr Eng, 2019, 73, 84-96

[21] Kumar P.A.R., Selvakumar S., Distributed denial of service attack detection using an ensemble of neural classifier. Comput Commun, 2011, 34(11), 1328-41

[22] Al-garadi M.A., Mohamed A., Al-ali A., Du X., Guizani M., A Survey of Machine and Deep Learning Methods for Internet of Things (IoT) Security, arXiv Prepr, 2018, 1-42

[23] Tan Z., Jamdagni A., He X., Nanda P., Liu RP., A system for denialof-service attack detection based on multivariate correlation analysis, IEEE Trans Parallel Distrib Syst, 2014, 25(2), 447-56

[24] Cui J., Wang M., Luo Y., Zhong H., DDoS detection and defense mechanism based on cognitive-inspired computing in SDN, Futur Gener Comput Syst, 2019, 97, 275-83

[25] Osanaiye O., Cai H., Choo K.R., Dehghantanha A., Xu Z., Dlodlo $M$., Ensemble-based multi-filter feature selection method for 
DDoS detection in cloud computing, J Wirel Commun Netw, 2016

[26] Prasad K.M., Reddy A.R.M. Rao K.V., BARTD: Bio-inspired anomaly based real time detection of under rated App-DDoS attack on web, J King Saud Univ - Comput Inf Sci, 2017

[27] Gavrilis D., Dermatas E., Real-time detection of distributed denialof-service attacks using RBF networks and statistical features, Comput Networks, 2005, 48(2), 235-45

[28] Du D., Li X., Li W., Chen R., Fei M., Wu L., ADMM-Based Distributed State Estimation of Smart Grid Under Data Deception and Denial of Service Attacks, IEEE Trans Syst Man Cybern Syst, 2019, 49(8), 1-14
[29] Dwivedi S., Vardhan M., Tripathi S., Kumar A., Implementation of adaptive scheme in evolutionary technique for anomaly-based intrusion detection, Evol Intell, 2020, 13(1), 103-17

[30] Shukla A.K., Building Effective Approach Toward Intrusion Detection Framework : Using Ensemble Feature Selection, Int J Inf Secur Priv, 2019, 13(3), 31-47

[31] Farid D.M., Zhang L., Rahman C.M., Hossain M.A., Strachan R., Hybrid decision tree and naïve Bayes classifiers for multi-class classification tasks, Expert Syst Appl, 2014, 41(4), 1937-46

[32] Tan Z., Detection of Denial-of-Service Attacks Based on Computer Vision Techniques, IEEE Trans Comput, 2013, 64, 1-14 